\title{
Calidad y nuevas tecnologías como ejes del proceso de modernización de la Administración pública: el enfoque integrado de la Escola Galega de Administración Pública
}

\author{
Moneyba González Medina \\ Escola Galega de Administración Pública \\ moneyba.gonzalez@gmail.com \\ Rocío Fentanes Fortes \\ Escola Galega de Administración Pública \\ rocio.fentanes.fortes@xunta.es
}

\author{
Belén Murillo Gorozarri \\ Ayuntamiento de San Sebastián de los Reyes \\ belen_m_g@hotmail.com \\ Carmen Voces López \\ Escola Galega de Administración Pública \\ carmen.voces.lopez@xunta.es
}

\begin{abstract}
Resumen
En la última década, se ha ido configurando un marco normativo y político que pone el acento en la introducción de sistemas de calidad y el uso intensivo de las TIC en la gestión pública, convirtiéndolas en dos líneas de transformación clave de los procesos de modernización administrativa en un contexto de gobernanza. Por ello, con el objetivo de adaptarse a las necesidades de una sociedad abierta y compleja, la Escola Galega de Administración Pública ha puesto en marcha una estrategia de modernización a partir de un enfoque integrado en el que las dimensiones de calidad e introducción de las nuevas tecnologías se refuerzan mutuamente, convirtiéndose en los ejes de cambio de su modelo de gestión. Así, se describirá cómo la combinación de estas dos dimensiones se encuentra presente en cada uno de los proyectos a través de los que la EGAP despliega dicha estrategia, contribuyendo, desde una orientación hacia la mejora continua, no sólo a la satisfacción de las expectativas de sus usuarios/as y a la provisión de servicios de máxima calidad, sino también a un proceso más amplio de transformación hacia una Administración pública más transparente, participativa, responsable y eficiente.
\end{abstract}

Palabras clave Administración electrónica, calidad, EFQM, evaluación de resultados, planificación estratégica, gestión pública, interoperabilidad.

\section{Quality and new technologies as axis of the process of modernization in the Public Administration: Integrated approach of the Galician School of Public Administration}

\begin{abstract}
Over the last decade a new political and regulatory framework has taken shape emphasizing the introduction of quality systems and intensive usage of ICT in the public management sector. These two concepts are key in the process of modernizing public administration within the context of governance. In response to an open society and it's complex needs, the Galician School of Public Administration has launched a new strategy to modernize management in the public sector. This strategy focuses on the alignment of new technologies and quality procedures that become the axis on which change management models are developed. It will be described how the combination of these two key concepts can be found in every single project launched by the EGAP. While focusing on continual improvement, these concepts not only aim at satisfying users' expectations and supplying services of the highest quality, but also have a grander scope of moving towards a more accountable, open and efficient Public Administration.
\end{abstract}

Keywords

E-administration, quality, EFQM, outcomes evaluation, strategic planning, public management, interoperability. 


\section{INTRODUCCIÓN}

En la última década, en la Comunidad Autónoma de Galicia se ha ido configurando un escenario normativo que ha promovido tanto la introducción de instrumentos de mejora continua como el empleo de las nuevas tecnologías en la gestión pública. Desde estos presupuestos, la Escola Galega de Administración Pública' (EGAP) ha querido aprovechar este contexto para poner en marcha una estrategia de modernización de los servicios que presta, basada en la implementación conjunta de ambas líneas de transformación.

De esta manera, la EGAP asumió el reto de diseñar una estrategia de modernización global, superadora de ejercicios aislados que, basándose en la calidad y en las nuevas tecnologías de la información y comunicación (TIC) y de acuerdo con las corrientes de modernización administrativa presentes en el contexto normativo estatal y autonómico, no sólo contribuyera a mejorar la gestión interna de la Escuela y la prestación de sus servicios, sino que se concibiese como un instrumento de innovación en la relación con las personas usuarias de estos, posibilitando nuevas pautas de interacción con las mismas.

Partiendo de un enfoque sistémico, que considera las organizaciones como un agregado de variables (objetivos, estructura, recursos humanos, tecnología y procedimientos administrativos) que modifican sus propias configuraciones y sus sistemas de interacción con el resto de las variables en función de las exigencias del entorno de la organización (Ramió, 1999: 266), el presente artículo analiza la incidencia de las variables "calidad" y "nuevas tecnologías" en el proceso de cambio organizativo de la Escola Galega de Administración Pública, a partir de la descripción de las distintas iniciativas y actuaciones que se están llevando a cabo en el marco de su proyecto integral de modernización. Así, este trabajo se plantea como un estudio de caso, destinado a describir las iniciativas que lo componen, poniendo el énfasis en la impronta de la calidad y las TIC en el proceso de cambio, tratando de aportar datos empíricos que pongan de manifiesto la importancia de desarrollar una estrategia combinada, basada en la calidad y en las nuevas tecnologías, en los procesos de reforma de las administraciones públicas.

Con este propósito, el análisis y la descripción de los proyectos e iniciativas desarrolladas en el marco de esta estrategia se presentan desde un triple enfoque que implica el análisis de la evolución de la propia organización, de los procesos de trabajo requeridos para su implementación y, por último, de los resultados alcanzados. Para ello, se parte de una metodología basada en el análisis documental de los informes y las memorias de los proyectos descritos, así como de la normativa aplicable; y en la observación directa del proceso de cambio de la organización objeto de análisis.

1 La EGAP es un organismo autónomo adscrito a la Consellería de Presidencia, Administracións Públicas e Xustiza (Ley 4/1987, de 27 de mayo, de creación de la EGAP, modificada por la Ley 10/1989, de 10 de julio), encargado de las tareas de selección y formación del personal al servicio de la Administración 94 pública gallega, así como del estudio y la difusión de la investigación en el ámbito de las ciencias de la Ad-
ministración. 
Asimismo, se toma como marco de referencia el proceso de modernización de la administración pública y el desarrollo normativo que, en materia de calidad y de servicios digitales, se ha producido tanto a nivel estatal como autonómico.

\section{ELEMENTOS CARACTERIZADORES DEL PROCESO DE MODERNIZACIÓN DE LA ADMINISTRACIÓN PÚBLICA}

Al analizar la evolución de la gestión pública, se observa que la calidad y el desarrollo de la administración electrónica constituyen dos líneas de transformación que han cobrado distinto protagonismo a medida que avanzan los procesos de modernización de la administración pública.

El dominio de la Nueva Gestión Pública, que marcó el auge de la calidad total (Total Quality Management), se sitúa en la década de los ochenta y principios de los noventa bajo la hegemonía de la agenda neoliberal. En este período, el énfasis en las "3 E” (Economía, Eficiencia y Eficacia) llevó a que la agenda de reformas estuviera dominada por la "ingeniería de procesos", la evaluación de los servicios y la satisfacción de los usuarios/as con el objetivo de compatibilizar la situación deficitaria de la administración con la prestación de servicios de calidad (Ballart y Ramió, 2000).

Los modelos de gestión basados en este paradigma tienen como objetivo último la búsqueda de la "calidad total" en la producción de los bienes, mercancías y servicios que produce la organización partiendo de la "orientación al cliente" como principio rector de todas sus actividades, introduciendo una nueva concepción general de la gestión (Ehrenberg y Stupak, 1994; Parrado y Ruiz, 1999; Bañón y Carrillo, 1996; Domínguez, 2004) basada en los siguientes elementos (Voces, Rodríguez y Caínzos, 2009: 14-15):

- Concepción holística de la calidad, que abarca toda la organización y todos los procesos que en ella tienen lugar y en cuya generación han de estar implicados todos los miembros de la organización.

- Vinculación entre calidad interna (hacia dentro de la propia organización) y externa (orientada al usuario o consumidor).

- Búsqueda de la satisfacción de las expectativas de los clientes al menor coste posible.

- Adopción de una visión corporativa en la que exista una única estrategia hacia un único fin: la calidad y la mejora constante.

- Impulso de la participación de los trabajadores en el objetivo global de la calidad; el trabajo en equipo y la toma de decisiones compartidas adquieren especial importancia. 
- Atribución de relevancia a los procesos a cuya mejora continua se deben encaminar de manera preferente los esfuerzos de los gestores.

- Establecimiento de sistemas de medición que permitan contar con datos objetivos sobre la marcha de los procesos, la calidad de los productos y el grado de cumplimiento de los objetivos fijados, información que será la base para la toma de decisiones estratégicas.

Hacia finales de los noventa y en la primera década del siglo XXI, con la revolución tecnológica, se fue consolidando una nueva dinámica orientada a la creación de entornos cooperativos para hacer frente a la complejidad de las demandas de las sociedades contemporáneas y a los nuevos retos derivados de la globalización. Así, empieza a hablarse del paradigma de la gobernanza (Kooiman, 2002; Mayntz, 2000; Cerrillo i Martínez, 2005; Prats i Catalá, 2005) para hacer referencia a un nuevo marco complejo de relaciones entre los agentes intervinientes en la toma de decisiones y en la prestación de los servicios públicos. En este contexto, se multiplican las experiencias que persiguen la puesta en marcha de fórmulas de colaboración, la comunicación e intercambio de información, así como la eliminación de barreras personales, temporales y espaciales en el ámbito de la prestación de servicios públicos. En el desarrollo de estas fórmulas, como se ha señalado, la revolución tecnológica ha desempeñado un papel clave: "Los medios electrónicos pueden contribuir decididamente a mejorar la gobernanza de nuestro tiempo. Desde esta perspectiva los medios electrónicos pueden ser utilizados no sólo para mejorar la eficacia administrativa sino también para contribuir a una mayor transparencia administrativa, una mejor participación ciudadana en las administraciones públicas y una más intensa rendición de cuentas" (Cerrillo i Martínez, 2010: 48).

En este escenario complejo, resulta fundamental para la gestión pública el desarrollo de instrumentos que permitan formular políticas sobre la base del conocimiento de la realidad (técnicas de diagnóstico), con altas dosis de creatividad y con visión estratégica, y que faciliten el trabajo con diferentes organizaciones, departamentos y servicios. Dicha formulación debe responder al enfoque de sistemas, con una idea clara de los actores implicados (y de sus recursos), las interconexiones y los objetivos a conseguir. En este sentido, el management deja de ser concebido principalmente como planificación y control para visualizarse ante todo como dirección, estrategia o política, o, mejor, como asunción de la responsabilidad por la acción colectiva (Prats i Catalá, 2005: 125). De este modo, los últimos treinta años de modernización administrativa pasan por tres fases inspiradas por distintas "ideas fuerza": la racionalidad burocrática, la calidad total y la interoperabilidad ${ }^{2}$, que sirven para sostener la tesis

2 Según el Real Decreto 4/2010, de 8 de enero, por el que se regula el Esquema Nacional de Interoperabilidad en el ámbito de la Administración Electrónica: “La interoperabilidad es la capacidad de los sistemas de información y de los procedimientos a los que éstos dan soporte de compartir datos y posibilitar el intercambio de información y conocimiento entre ellos. Resulta necesaria para la cooperación, el desarrollo, la integración y la prestación de servicios conjuntos por las Administraciones públicas; para la 
del paso de la administración (burocracia) a la gerencia (management) y de la gerencia a la gobernanza (governance) (Prats i Catalá, 2005).

\section{El contexto normativo de la reforma administrativa}

En consonancia con lo anterior, la producción normativa en el contexto de la reforma administrativa se ha orientado claramente en los últimos años hacia dos temáticas fundamentales: el desarrollo de sistemas de calidad en la gestión y la introducción de las nuevas tecnologías de la información y comunicación en las organizaciones públicas. Estas temáticas se corresponden con dos momentos de desarrollo normativo bien diferenciados, tal y como puede observarse en el Cuadro 1.

El primer período, caracterizado por la promoción de herramientas de calidad en la gestión pública (normas ISO, EFQM, cartas de servicio, etc.), abarcaría los años 2005-2008. El segundo, que arrancaría con la aprobación de la Ley 11/2007, de 22 de junio, de acceso electrónico de los ciudadanos a los Servicios Públicos (LAECSP), marca un nuevo hito al reconocer, por primera vez en nuestro ordenamiento, el derecho de la ciudadanía a relacionarse electrónicamente con la Administración: "Con la aprobación de la LAECSP, se abre ahora una nueva oportunidad para una segunda generación de normas de las Comunidades Autónomas que regulen la administración electrónica y que impulsen a través del derecho un proceso de transformación de las administraciones públicas autonómicas basado en el uso intensivo de los medios electrónicos que facilite una administración pública no sólo más eficaz, sino también más transparente, más participativa, más responsable y en red" (Cerrillo i Martínez, 2010: 52). Así, puede observarse que la apuesta por las TIC y el uso de los medios telemáticos en la administración empieza a concebirse como un complemento de los instrumentos de calidad, es decir, como un medio que contribuye a reforzar la filosofía de la mejora continua³.

No obstante, la introducción de las TIC en las administraciones públicas de las Comunidades Autónomas, con el desarrollo de la LAECSP, se enfrenta a diferentes retos que afectan a la organización interna de la propia Administración y a la manera en que la ciudadanía se relaciona con ella (Cerrillo i Martínez, 2011: 34), poniéndose de manifiesto dificultades en la puesta en marcha de los servicios digitales previstos en

ejecución de las diversas políticas públicas; para la realización de diferentes principios y derechos; para la transferencia de tecnología y la reutilización de aplicaciones en beneficio de una mejor eficiencia; para la cooperación entre diferentes aplicaciones que habiliten nuevos servicios; todo ello facilitando el desarrollo de la administración electrónica y de la sociedad de la información."

3 Resulta ilustrativo el preámbulo del Decreto 198/2010, de 2 de diciembre, por el que se regula el desarrollo de la Administración electrónica en la Xunta de Galicia y en las entidades de ella dependientes, en el que se señala que: "El Gobierno de la Xunta de Galicia tiene dentro de sus ejes de actuación impulsar la modernización y la innovación tecnológica al objeto de potenciar la mejora de la calidad de servicio ofrecido a los ciudadanos, convirtiendo a la Xunta de Galicia en un modelo que sea referente para el desarrollo de la Administración electrónica en Galicia y en todos sus ámbitos." 
la ley. En este sentido, en el Cuadro 2 se muestra el porcentaje de Comunidades Autónomas que han implementado las distintas actuaciones previstas en la legislación.

\section{CUADRO 1}

Marco normativo de modernización de la Administración pública

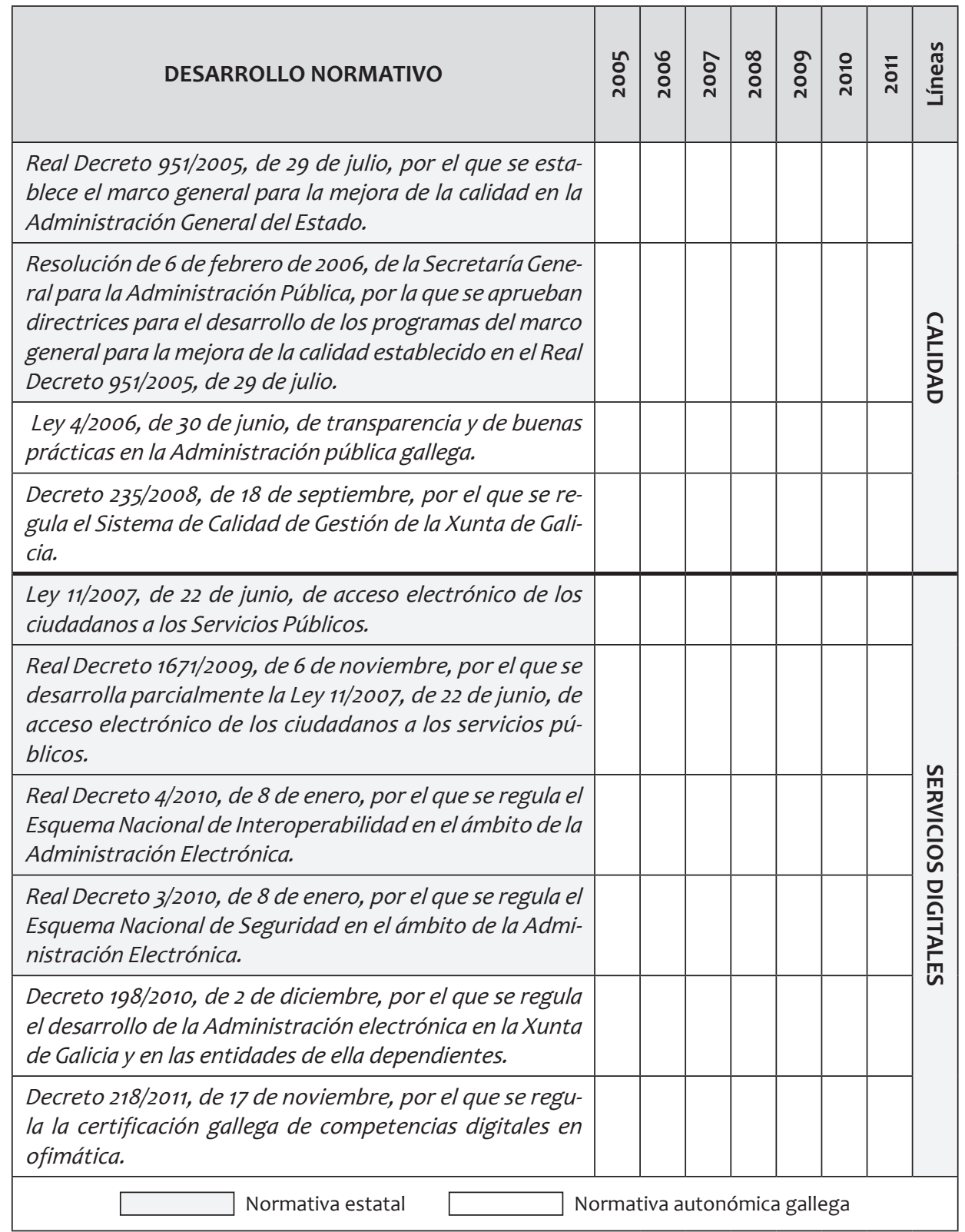

98 Fuente: Elaboración propia. 
CUADRO 2

Desarrollo de la administración electrónica a nivel autonómico

\begin{tabular}{|l|c|}
\hline \multicolumn{1}{|c|}{ SERVICIOS ELECTRÓNICOS A LA CIUDADANÍA } & $\mathbf{2 0 0 9}$ \\
\hline Inicio de procedimiento & $94,00 \%$ \\
\hline Sede electrónica & $33,00 \%$ \\
\hline Registro electrónico & $94,00 \%$ \\
\hline Pago electrónico & $93,00 \%$ \\
\hline Comunicaciones Administrativas electrónicas & $100,00 \%$ \\
\hline Constancia de comunicaciones & $94,00 \%$ \\
\hline Notificaciones electrónicas & $63,00 \%$ \\
\hline Portal web & $100,00 \%$ \\
\hline No aportar documentos que ya obren en poder de la Administración & $69,00 \%$ \\
\hline Copias electrónicas & $25,00 \%$ \\
\hline Archivo de expedientes electrónicos & $56,00 \%$ \\
\hline Consulta electrónica de tramitaciones & $75,00 \%$ \\
\hline Canales de comunicación & $73,00 \%$ \\
\hline
\end{tabular}

Fuente: Elaboración propia a partir de los datos extraídos del Informe CAE 2009. La Administración Electrónica en las Comunidades Autónomas'.

\section{La planificación estratégica en la Xunta de Galicia: calidad y TIC como ejes vertebradores}

En este escenario normativo, la Xunta de Galicia impulsa varios instrumentos de planificación entre los que destaca el Plan Estratéxico de Galicia 2010-20145 (PEG 20102014) $)^{6}$, que define sus líneas de acción atendiendo a dos directrices de desarrollo: la cohesión y la gobernanza.

$4 \quad$ El número de Comunidades Autónomas que participan en el cálculo del porcentaje para cada indicador son: Registro electrónico y comunicaciones, administraciones electrónicas (16 CCAA) - Inicio procedimientos, pago electrónico, constancia, comunicaciones y portal web (15 CCAA) - No aportar documentos y consulta electrónica, tramitación (13 CCAA) - Notificaciones electrónicas y archivo expedientes electrónicos (12 CCAA) - Canales comunicación (11 CCAA) - Copias electrónicas (10 CCAA) - Sede electrónica (9 (CAA).

5 Plan Estratéxico de Galicia 2010-2014. Disponible en: <http://www.conselleriadefacenda.es/ plan-estratexico/pdfs/plan_estratexico_version_web.pdf> (Consulta: 9 de agosto de 2011).

6 Este hecho conllevó la integración dē las actuaciones emprendidas por la EGAP en una estrategia más amplia que exige una estrecha colaboración con otras entidades de la Xunta de Galicia, especialmente con la Secretaría Xeral de Modernización e Innovación Tecnolóxica y la Dirección Xeral de Avaliación e Reforma Administrativa de la Consellería de Presidencia, Administracións públicas e Xustiza. 


\section{FIGURA 1.}

Directrices de desarrollo del PEG 2010-2014

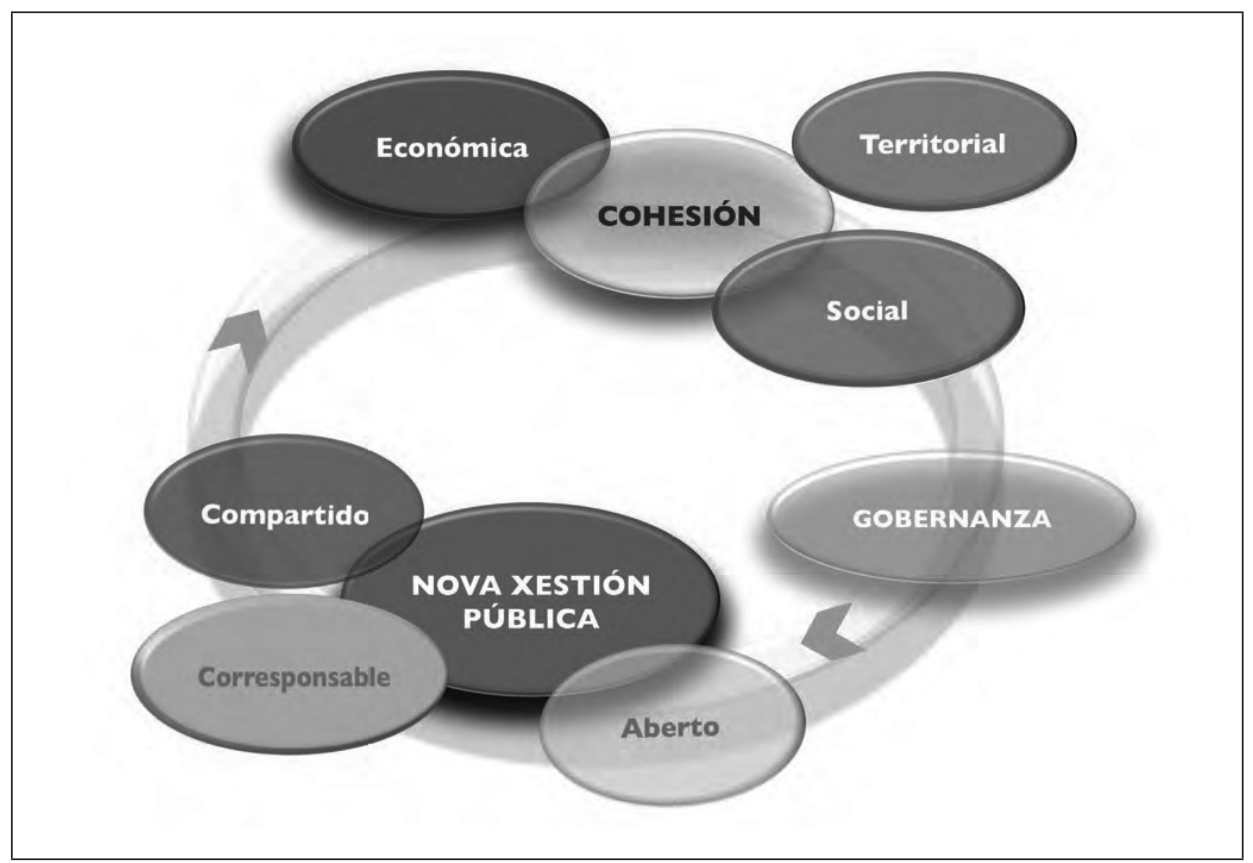

Fuente: Plan Estratéxico de Galicia 2010-2014.

El plan apuesta por un modelo de gobernanza abierto, corresponsable y compartido, que parte de la convicción de que para adaptarse a los nuevos modelos de relación es preciso un cambio normativo, organizativo y tecnológico que fomente y facilite la colaboración entre las administraciones públicas. Esta colaboración exige lo siguiente: que la ciudadanía perciba unos servicios públicos homogéneos, garantizándose el mismo nivel de calidad, acceso y servicio para todas las personas (abierto); que las administraciones se corresponsabilicen y cooperen, implantando los mismos estándares y procedimientos de actuación (corresponsable); y que se garantice la reutilización de las inversiones en sistemas de información, haciendo frente al reto de la interoperabilidad (compartido).

Teniendo presente este contexto, desde principios del año 2010, tras casi veinticinco años de historia, la Escola Galega de Administración Pública se propone iniciar un proceso de cambio organizativo y de gestión que persigue su adaptación a las necesidades de una sociedad abierta7 y compleja. Por este motivo, aunque la labor de la

7 Para Henri Bergson (1845 - 1941) las sociedades abiertas son aquellas que tienen gobiernos tolerantes, que responden a las demandas de transparencia y flexibilidad de la ciudadanía. En estas sociedades se asume que el conocimiento es patrimonio de todos (Possenti, 1985: 283-290). 
Escuela se orienta a la formación del personal de la administración pública gallega, también se ha considerado necesario extender su misión a la sociedad en su conjunto y convertirla en una “organización del conocimiento". De este modo, el impacto de su misión formativa, con la ayuda de las nuevas tecnologías, situaría a la organización más cerca de la visión de una EGAP moderna, que incorpora las nuevas tecnologías en la gestión del conocimiento y que presta sus servicios con la máxima calidad. Una EGAP abierta, de todos y para todos. No obstante, esta visión planteó el desafío de emprender toda una serie de reformas para prestar servicios de máxima calidad en un contexto de austeridad presupuestaria.

Para abordar estas reformas, la EGAP decidió combinar dos líneas de transformación clave para mejorar y adaptar sus servicios a los cambios que se estaban produciendo en la sociedad: la apuesta por la calidad y por los servicios digitales, aplicando un enfoque integrado a una estrategia alineada con los objetivos del PEG 2010-2014 de "Modernizar y promover la aplicación de las TIC en los ámbitos productivos, social y servicios públicos (E3: Economía del conocimiento, OE 3.2) y de Lograr una Administración moderna y próxima, optimizando los recursos materiales y humanos mediante la implantación de nuevos sistemas y procedimientos de gestión y generalizando el empleo de las TIC en todos sus ámbitos de actuación" (E5: Administración Austera, Eficiente y Próxima al Ciudadano, OE 5.1).

Teniendo en cuenta lo anterior, las líneas estratégicas en las que se ha basado la acción transformadora de la organización y de los servicios que presta la EGAP son las siguientes:

a) Compromiso con la modernización e innovación tecnológica en la administración pública gallega, mediante acciones como el incremento de la oferta formativa en la modalidad de teleformación.

b) Más formación práctica y en línea, con las necesidades reales de la administración y de los empleados públicos.

c) Más descentralización de la formación, con una actividad cada vez mayor en todo el territorio gallego, para que los empleados públicos puedan acceder a la formación en las mejores condiciones y conciliar los tiempos.

d) Transparencia y compromiso con los usuarios/as de la EGAP -administración, empleados públicos y docentes- mediante la evaluación continua, permanente y sistemática de la calidad y de la satisfacción con la formación.

e) Excelencia en la prestación de los servicios como eje transversal de la actuación de la Escuela, basada en herramientas y marcos de referencia como el Modelo Europeo EFQM de Excelencia, la norma UNE-EN ISO 9001:2008 o la metodología de Carta de servicios de la Xunta de Galicia. 
f) Eficiencia en la gestión presupuestaria y austeridad, ofreciendo formación de calidad a un menor coste gracias a la introducción de mejoras en la gestión y al uso de las tecnologías.

\section{LA ESTRATEGIA DE MODERNIZACIÓN DE LA EGAP}

El desarrollo de las líneas estratégicas de la EGAP se está llevando a cabo a través de diversos proyectos cuyo denominador común es el uso intensivo de las TIC y la introducción de mejoras en la gestión derivadas de la implantación de sistemas de calidad. Como se podrá observar, ambos elementos se combinan en cada proyecto para implementar una estrategia integrada con la que la Escuela espera lograr el objetivo de prestar servicios de calidad, de acuerdo con las demandas de una sociedad cambiante y compleja.

\section{Promoción de la calidad: EFQM, carta de servicios, SIAC e implantación de la norma UNE-EN ISO 9001:2008}

En materia de gestión de la calidad, existen dos grandes tendencias que se materializan en la apuesta por la implantación de herramientas de calidad basadas en la ISO 9000 o normas similares, que están enfocadas fundamentalmente en el sistema productivo de la organización, o en la apuesta por la calidad total orientada a la mejora de la gestión y de los resultados de la organización, como es el caso del Modelo EFQM (Fundación Europea para la Gestión de Calidad) ${ }^{8}$ de Excelencia (Membrado, 1999: 7). El Modelo EFQM establece un marco constituido por un conjunto de factores o criterios interrelacionados entre sí que definen a una organización teóricamente excelente, capaz de lograr y mantener los mejores resultados posibles. La aplicación de este modelo a una organización permite realizar una evaluación de los diferentes elementos que la integran (valores, modelo de gestión, procesos, resultados, etc.) y realizar comparativas, poniendo de manifiesto los puntos fuertes y las áreas de mejora de la organización evaluada y su situación respecto al nivel teórico de excelencia definido por el modelo. Desde esta perspectiva, el "Modelo EFQM de Excelencia" puede ser considerado como una herramienta de identificación de oportunidades de mejora, pero con la ventaja de que al utilizar un referente permite realizar una evaluación global, en profundidad y sistemática, sin descuidar ninguno de los aspectos importantes que pueden determinar la calidad de la organización (Maderuelo, 2002: 633).

La EGAP apostó por el Modelo EFQM de Excelencia (versión 2010) como marco global de actuación para llevar a cabo su estrategia modernizadora, debido a que favorece la innovación y el aprendizaje de la organización, permitiéndole anticiparse a

8 El Modelo EFQM, cuyas siglas hacen referencia a la European Foundation for Quality Management tuvo sus orígenes en 1988. Véase <http://www.efqm.org>. 
los cambios del entorno mediante un sistema de autoevaluación de cada uno de los aspectos clave en la gestión de la Escuela, relacionando los resultados obtenidos con los agentes facilitadores.

FIGURA 2

Modelo EFQM de Excelencia 2010

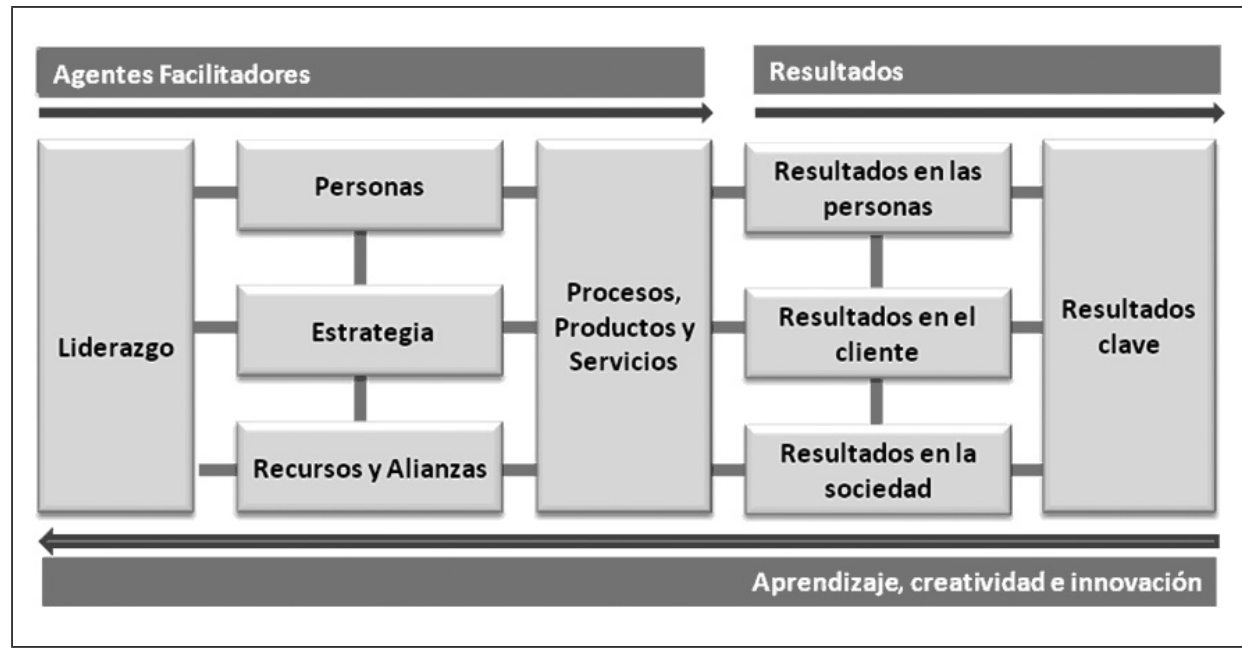

Fuente: Elaboración propia a partir del Modelo EFQM.

Además de las acciones de mejora prioritarias definidas como resultado de la evaluación -carta de servicios, documentación y mejora de los procesos (UNE-EN ISO 9001:2008) y evaluación del clima laboral-, la EGAP ha ido desarrollando otros proyectos que contribuyen al refuerzo de su estrategia de modernización, como la reforma de su estructura orgánica, con la que se consigue la adaptación de la organización al ciclo PDCA ${ }^{9}$ de mejora continua, o el desarrollo de un Sistema integral de evaluación de la calidad de la oferta formativa de la Escuela (SIAC).

\section{- Implantación del Modelo EFQM en la EGAP}

El principal objetivo del proyecto de Autoevaluación de la gestión de la EGAP según el Modelo EFQM de Excelencia era obtener un diagnóstico inicial de la organización que posibilitase la elaboración de un plan de mejora y la articulación de medidas concretas para llevar a cabo las reformas. Los principales hitos en el marco de este proyecto se concretan en el siguiente gráfico:

9 También conocido como círculo de Deming, el ciclo PDCA de la mejora continua hace referencia a las siglas Plan-Planificar-, Do-Hacer-, Check-Verificar-y Act-Actuar-. 
FIGURA 3

Implementación del proyecto de Autoevaluación de la gestión de la EGAP según el Modelo EFQM 2010

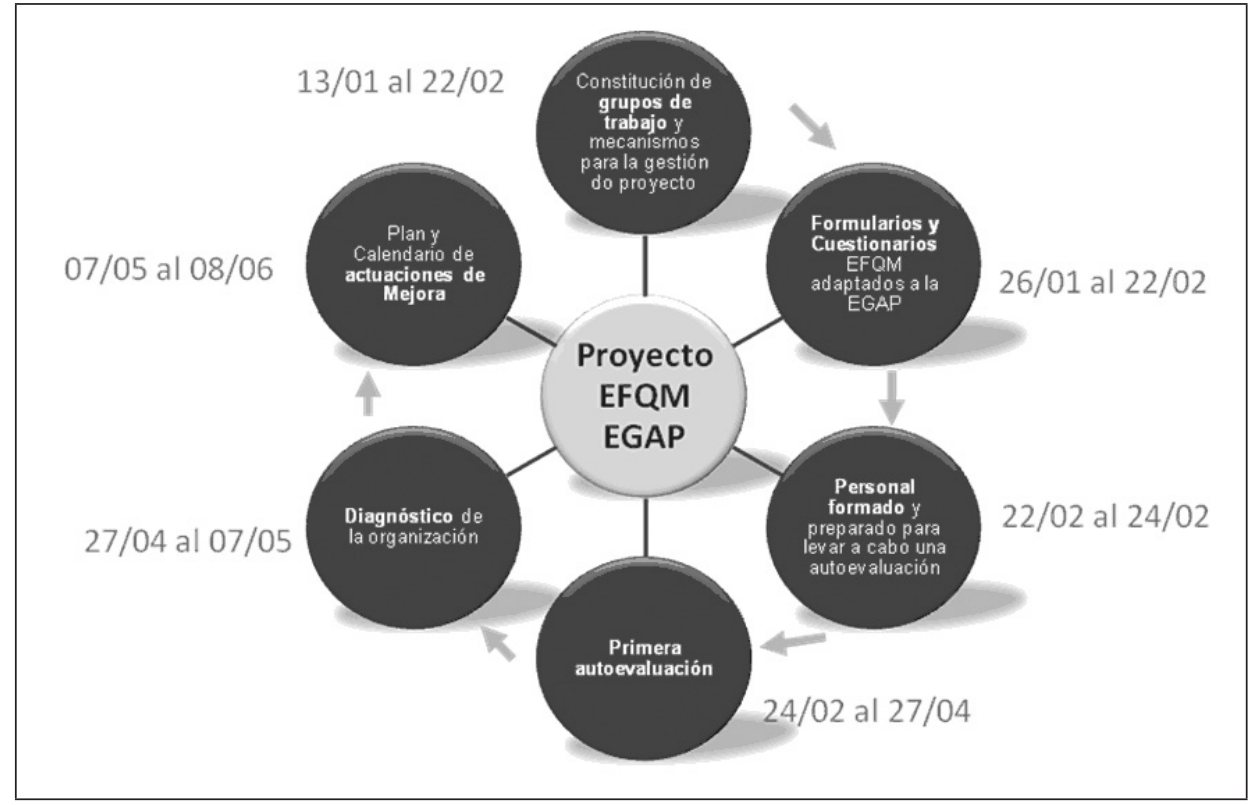

Fuente: Unidad de Investigación, Evaluación y Calidad de la EGAP.

La puesta en marcha del proyecto requirió la constitución de un grupo de trabajo ad hoc integrado por personal de las distintas áreas organizativas y niveles jerárquicos de la EGAP. Asimismo, se emprendió la recopilación de la documentación de la Escuela con impacto en las distintas áreas de análisis del modelo y se pusieron en práctica los planes de comunicación y participación, basados en el uso de la Intranet, el correo electrónico y el desarrollo de sesiones informativas presenciales.

A lo largo de dos meses se celebraron las reuniones de consenso semanales destinadas a la evaluación de cada uno de los criterios y subcriterios del modelo. La participación del personal en los grupos de mejora y la necesidad de consensuar un diagnóstico en cada una de las sesiones supuso un valor en sí mismo, al fomentar el intercambio de ideas, el debate y la defensa de posiciones.

El aprendizaje adquirido por la EGAP tras el proceso ha resultado clave no sólo para la definición de las líneas de actuación sino también para su implementación, gracias a la participación activa del personal de la Escuela que ha contribuido a la apropiación del proyecto de mejora. Fruto del proceso, fueron identificados los puntos fuertes y débiles de la EGAP que sirvieron de base para la elaboración del "Plan de mejora" en el que se definieron y priorizaron las medidas que la organización debía poner en marcha en el marco de su estrategia de modernización. 
Entre las fortalezas identificadas en este proceso destaca la promoción de acciones de reestructuración organizativa orientadas a alcanzar una mayor funcionalidad y eficiencia, a pesar de la reducción del presupuesto, consiguiendo aumentar el número de servicios prestados en los últimos años ${ }^{10}$. A partir de estas consideraciones se llevó a cabo una reestructuración interna de la Escuela mediante el Decreto 175/2010, de 14 de octubre, por el que se establece la estructura orgánica de la Escola Galega de Administración Pública11, con el objetivo de evitar la duplicidad de atribuciones de los servicios y proceder con mayor racionalidad y eficiencia en la gestión de los recursos a la hora de planificar y gestionar las actividades formativas. Esta reestructuración se concretó con la creación del Servicio de Programación de Actividades de Formación y el Servicio de Gestión de Actividades de Formación y Selección, modificando la anterior configuración de los servicios de formación que respondía al tipo de destinatarios -administración autonómica, por una parte, y administración local y otras administraciones, por otra- y en la que cada uno desarrollaba las funciones de planificación y de gestión en su área respectiva.

Además, por medio de este Decreto se crea la Unidad de Investigación, Evaluación y Calidad que gestiona, establece y mantiene procedimientos de gestión interna para proporcionar a las distintas Unidades y Servicios los instrumentos necesarios para una mejora continua en la calidad de los servicios prestados por la Escuela y propone al Servicio de Programación de las Actividades de Formación los procedimientos que permiten evaluar la calidad de las actividades formativas desarrolladas por la Escuela. La presencia de esta unidad garantiza la evaluación permanente de la actividad de la Escuela, así como la disponibilidad de la información necesaria para poner en marcha los ciclos de mejora continua en la prestación de sus servicios.

En este proceso de cambio de estructura orgánica, merece especial mención el hecho de que fuese precedido de una consulta al personal de la Escuela en la que se preguntaba sobre la valoración de la propuesta de cambio, en términos generales y para cada una de las modificaciones concretas planteadas. La tasa de respuesta alcanzó el 83\% del total de cuestionarios repartidos. En líneas generales, los resultados revelaron un importante consenso en relación con la idea que subyace al nuevo modelo -eficacia, eficiencia y un mejor servicio a los usuarios/as- y una acogida favorable al contenido concreto de la propuesta de cambio de estructura. En lo que respecta a las potenciales ventajas del cambio, el personal destacó especialmente las relativas a la mejora de la coordinación entre los distintos servicios, la reducción de las duplicidades en el trabajo y la ruptura con las inercias de la organización ${ }^{12}$.

Por otra parte, el resultado de la autoevaluación según el Modelo EFQM puso de manifiesto que uno de los aspectos más críticos hacía referencia a la necesidad de

10 Conclusiones extraídas del Informe de diagnóstico del proyecto Autoevaluación de la gestión de la EGAP según el Modelo EFQM de Excelencia (2010: 70-71).

11 Diario Oficial de Galicia de 25 de octubre de 2010.

12 Informe interno de la Unidad de Investigación, Evaluación y Calidad relativo a la Encuesta sobre la modificación de la Estructura Orgánica de la EGAP. Documento sin publicar. 
contar con mecanismos que garantizasen el seguimiento continuo de las acciones desarrolladas y el análisis de los resultados mediante un sistema de indicadores que permitiera evaluar el rendimiento de la organización. Por ello, la EGAP puso en marcha dos proyectos a fin de subsanar esta situación: la elaboración de la Carta de servicios y el establecimiento de un Sistema integral de evaluación de la calidad de la oferta formativa de la Escuela (SIAC). Además, quedó patente la importancia de sistematizar y documentar los procesos estratégicos, operativos y de apoyo de la organización y definir un mapa de procesos de la EGAP, actuaciones que la Escuela desarrolló en el marco del proyecto de implantación de la norma UNE-EN ISO 9001:2008, con el que la organización está llevando a cabo una mejora integral de los mismos. Asimismo, atendiendo al criterio "personas" del Modelo EFQM"13, la autoevaluación reveló la inexistencia de mecanismos para analizar la satisfacción de empleadas y empleados, por lo que la Escuela decidió poner en marcha diversas iniciativas en este ámbito centradas en promover la participación del personal en la toma de decisiones -como la encuesta sobre la nueva estructura orgánica y la encuesta sobre los compromisos de la Carta de servicios de la EGAP-, y conocer su grado de satisfacción dentro de la organización -como el diseño de una encuesta de clima laboral-.

Con la implantación de este modelo, la EGAP persigue el reconocimiento de la calidad de su gestión con el Sello de Excelencia "Compromiso hacia la excelencia en la gestión”, para lo que es preciso el despliegue y seguimiento de las acciones prioritarias recogidas en el Plan de mejora que serán descritas a continuación.

\section{- Carta de servicios de la EGAP}

Según la Guía descriptiva de elaboración de Cartas de Servicios en la Xunta de Galicia (2008: 9): "El actual reto de las administraciones públicas es conseguir la satisfacción de las necesidades y expectativas de los ciudadanos, adaptándose a la nueva realidad económica y social, propiciando un modelo de Administración caracterizada por la transparencia y la asunción de compromisos y haciendo públicos los niveles concretos de calidad comprometidos en la prestación de sus servicios, así como evaluando el grado de cumplimiento de dichos compromisos. Por otro lado, no puede obviarse que la nueva realidad socioeconómica está especialmente marcada por el avance y el uso de las nuevas tecnologías de la información y comunicación, obligando a las organizaciones públicas a reformular sus parámetros de organización, funcionamiento y buena gestión."

Desde estos presupuestos, en el marco del Plan de mejora del EFQM, la EGAP puso en marcha un proyecto destinado a la elaboración de una Carta de servicios, siguiendo la metodología establecida por el Decreto 117/2008, de 22 de mayo, por el que se regulan las cartas de servicios de la Xunta de Galicia y el Observatorio de la

13 Según el criterio "personas" del Modelo EFQM, una organización excelente es aquella que dispone de las personas más idóneas para el desempeño del trabajo y en la que estas conocen sus tareas y cuentan con los medios adecuados para desempeñarlas, alineando los objetivos personales de los trabajadores con los de la organización. 
Calidad y de la Administración Electrónica de Galicia, de acuerdo con el marco general establecido en la Ley $4 / 2006$, de 30 de junio, de transparencia y buenas prácticas en la Administración pública gallega, que hace referencia en su artículo 6 a las cartas de servicios. La Escuela optó por elaborar una carta de servicios de nivel auditado que implica un alto rigor metodológico al establecer no sólo requisitos de producto final sino también del proceso de elaboración ${ }^{14}$, entre los que destaca la necesidad de realizar un estudio de expectativas de los usuarios/as sobre la prestación y el nivel de calidad de los servicios; la evaluación y cuantificación, con datos objetivos y verificables, de los niveles reales de prestación/gestión de los mismos y el contraste de esos niveles reales con los estándares de calidad esperados por los destinatarios de los servicios; y la identificación y definición de las acciones, proyectos o planes de mejora a implantar a medio o largo plazo en relación con los servicios objeto de la carta, para poder incorporar en el futuro nuevos compromisos de calidad.

Durante el mes de septiembre de 2010 se llevaron a cabo las acciones de lanzamiento del proyecto, esto es, la comunicación oficial de la iniciativa a la Dirección Xeral de Avaliación e Reforma Administrativa (DXARA) y la información al personal de la organización, la constitución del equipo de trabajo del proyecto -que se encuentra integrado por personal del equipo directivo y por miembros del área de calidad - y la formación específica del equipo de trabajo.

Las fases de mayor relevancia en el desarrollo del proyecto fueron las relativas a la identificación y análisis de los servicios y al establecimiento de los compromisos de calidad que concluyeron con la identificación de un total de veintidós servicios y la incorporación a la carta de cuarenta y tres compromisos, en cuya definición participaron los tres agentes clave de la Escuela (personal gestor de la organización, alumnado y docentes), lo que facilita la alineación de las expectativas de los usuarios/as con la misión y visión de la organización.

En el desarrollo de ambas fases, tres han sido las consignas: el desarrollo de mecanismos de participación y comunicación, la orientación al usuario/a y el aprovechamiento de las nuevas tecnologías. Así, por ejemplo, para la detección de expectativas de los usuarios se analizó la información extraída de los "Cuestionarios para la evaluación de acciones formativas de la EGAP” (encuestas de satisfacción) y de las "Encuestas de detección de expectativas de los usuarios/as", específicamente diseñadas para el proyecto de carta de servicios, que se realizaron por vía electrónica.

El tratamiento de la información obtenida de las encuestas de satisfacción de los alumnos/as que participaron en alguna de las actividades de la Escuela en el año 2010 permitió obtener no sólo información cuantitativa, sino también cualitativa, mediante el análisis de las observaciones manifestadas en dichas encuestas ${ }^{15}$ (pregunta

14 Anexo II del Decreto 117/2008. Requisitos mínimos de proceso metodológico para la categoría carta de servicios auditada.

15 La información cuantitativa obtenida resultó del análisis de un total de 1.534 encuestas relativas a 108 actividades de formación desarrolladas por la EGAP entre el 1 de enero y el 30 de septiembre de 2010, 
abierta). Esta información se completó con la recabada a través de dos encuestas on line de detección de expectativas realizadas en noviembre de 2010, para cuya realización se utilizó una aplicación de código abierto, destinadas a conocer lo que los usuarios/as esperan de la EGAP.

\section{FIGURA 4}

Proceso de gestión de las cartas de servicios de la Xunta de Galicia

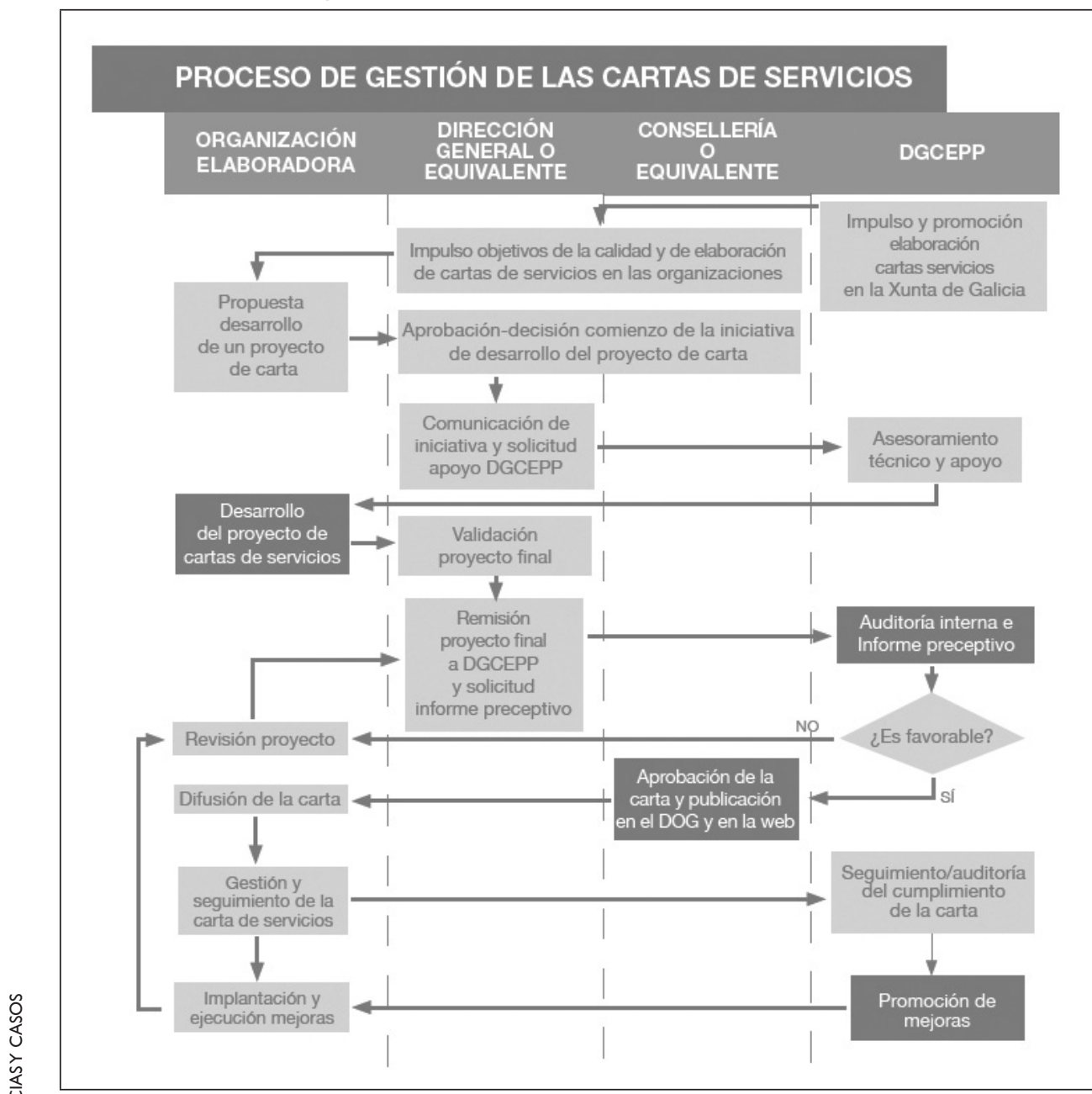

Fuente: Guía descriptiva de elaboración de Cartas de Servicios en la Xunta de Galicia.

siendo evaluados 244 docentes. En cuanto a la información cualitativa, se obtuvo del análisis de un total de 1.643 observaciones efectuadas por los alumnos/as -577 observaciones corresponden a formación presencial y 1.066 a actividades de teleformación- en relación a 169 actividades ( 98 presenciales y 71 de teleformación) desarrolladas en el mismo período, siendo cada una de las observaciones sometida a sucesivas recodificaciones mediante un procedimiento de aproximaciones sucesivas. 
En el diseño de la encuesta, se efectuó un muestreo aleatorio estratificado según el tipo de destinatario de la formación (personal de la administración autonómica, local y de justicia de la Comunidad Autónoma de Galicia) y se solicitó la colaboración de 3.982 personas (nivel de confianza del 95\%, p = q y error máximo del $\pm 2 \%$ para el conjunto de la muestra). Participaron en el estudio 1.256 personas lo que supone una tasa de respuesta del $32 \%$. En esta misma fase del proceso también se contó con el conjunto de docentes que habían impartido formación en la EGAP y de los 608 contactados obtuvimos respuesta de 198 (32,7\%). En la consulta, se preguntaba por sus opiniones y expectativas concretas en relación a cada uno de los servicios identificados, además de solicitarles que valorasen la importancia que les otorgaban con la finalidad de incorporar la perspectiva de alumnado y docentes, con carácter complementario a la opinión del personal gestor de la Escuela, en la priorización de los servicios. El hecho de contar también con las valoraciones del alumnado y los docentes a la hora de priorizar los servicios representa una mejora con respecto al diseño metodológico establecido, que contribuye a reforzar la presencia de los usuarios/as en el proceso de elaboración de la carta. La información obtenida, relativa a las necesidades y expectativas de los participantes en la acción formativa de la Escuela y de los docentes, fue utilizada como base para la determinación de los estándares de calidad y el establecimiento de los compromisos.

Como resultado de este proceso, la EGAP asumió un total de cuarenta y tres compromisos de calidad en la prestación de sus servicios entre los que destacan aquellos relacionados con la prestación de servicios digitales, poniendo así de manifiesto la doble dimensión de la estrategia modernizadora emprendida por la Escuela que incorpora las nuevas tecnologías como un eje transversal en los compromisos de calidad, presente tanto en la prestación como en la propia configuración de los servicios.

A este respecto, podemos mencionar los siguientes compromisos: la incorporación de un porcentaje mínimo de actividades formativas en la modalidad de teleformación, la edición y distribución de las publicaciones en soporte electrónico, la difusión a través de la página web de todo el material audiovisual resultante de las actividades divulgativas desarrolladas por la EGAP, el acceso a través de la página web de la Escuela a los temarios para los procesos selectivos convocados por la Xunta de Galicia y la realización, conjuntamente con Secretaría Xeral de Modernización e Innovación Tecnolóxica (SXMIT), de una convocatoria anual orientada a la certificación de competencias en el ámbito de las TIC. Asimismo, se contempla la inscripción y matrícula telemática en las actividades formativas convocadas por la EGAP, la posibilidad de consultar el estado de las solicitudes para participar en una actividad formativa mediante el enlace "Zona de matrícula" de la página web de la EGAP o el impulso de la interoperabilidad entre los distintos servicios, a través del intercambio de datos e información con la Dirección Xeral da Función Pública.

El proyecto de carta de servicios, íntegramente desarrollado por el personal de la EGAP, ha sido aprobado a nivel interno por la Escuela y se encuentra a la espera del 
informe preceptivo favorable de la DXARA, que es requisito previo para su publicación en el Diario Oficial de Galicia. No obstante, la EGAP ya ha logrado integrar los compromisos de la Carta en el desarrollo de su actividad cotidiana y en la prestación de sus servicios.

\section{— Sistema Integral de Evaluación de la Calidad: SIAC}

Otro proyecto estratégico ha sido el diseño y puesta en marcha de un Sistema Integral de Avaliación da Calidade da formación (SIAC) destinado a conocer los niveles reales de satisfacción ${ }^{16}$ con la oferta formativa y disponer de información para la planificación de actividades, que ha permitido institucionalizar un sistema formal y especializado de evaluación en el seno de la EGAP.

A través del SIAC, la evaluación, además de formar parte de los procesos operativos de la Escuela -desde la vertiente de administración y tratamiento de las encuestas-, se incluye también en la dimensión estratégica de la organización -en el nivel de los procesos relativos a la calidad, planificación de objetivos y definición y gestión de alianzas estratégicas- al suministrar información para la planificación de las actuaciones y el diseño de los planes formativos. Dicha información, en un esfuerzo por consolidar una cultura evaluativa y en aras de una mayor transparencia, se pone a disposición de los agentes que planifican la formación y de todo el personal de la EGAP. Asimismo, cualquier persona interesada en conocer la valoración de una actividad formativa en la que haya participado, podrá también tener acceso a los resultados de dicha evaluación.

El sistema de evaluación desarrollado, cuyo diseño, coordinación e implementación corresponde a la Unidad de Investigación, Evaluación y Calidad, se caracteriza por asumir una perspectiva integral al incorporar la opinión de los distintos agentes participantes en el proceso formativo (alumnado, personal docente y dinamizadores/ as de las acciones de teleformación -y tomar en consideración las peculiaridades de las diversas modalidades de formación- presencial y en línea). De este modo, proporciona datos de satisfacción del personal de las distintas administraciones -administración autonómica, local y de justicia-, con respecto a la formación presencial y la teleformación; valorándose, en el caso de la formación presencial, parámetros relacionados con la organización, contenidos, medios técnicos y materiales, aplicabilidad de los conocimientos al puesto de trabajo y docentes que intervienen. En el caso de la teleformación, además se evalúan cuestiones específicas de esta modalidad que hacen referencia a los aspectos técnicos y la resolución de problemas y a la posibilidad de autoorganización del proceso de aprendizaje y la flexibilidad para abordar los contenidos.

16 Si bien la Escuela ya utilizaba con anterioridad encuestas para evaluar la satisfacción con las actividades formativas, lo cierto es que la evaluación no presentaba un carácter sistemático ni se encontra-

110 ba integrada en el proceso formativo de la Escuela. 
La evaluación que realizan los docentes al finalizar la actividad formativa incluye cuestiones diversas orientadas a la valoración general del curso -puntuación global, relación entre los contenidos y la duración, nivel de cumplimiento de los objetivos marcados al comienzo del curso, motivación y participación de los asistentes-, así como una autoevaluación y también cuestiones generales destinadas a realizar una valoración de la EGAP.

Es importante señalar que el SIAC está incorporado en la Carta de servicios de la EGAP, y que a través de él se lleva a la práctica el cumplimiento de los cinco compromisos de calidad del servicio de evaluación que hacen referencia, entre otras cuestiones, a la evaluación del 100\% de las actividades formativas, a que la Escuela tenga en cuenta los resultados de la misma, al plazo de respuesta ante la solicitud de los interesados en conocer los datos de la evaluación, a la transparencia y a la difusión de los resultados a través de la página web ${ }^{17}$.

Por otra parte, con el objeto de continuar avanzando en la digitalización del sistema de evaluación, la Escuela ha aprovechado el potencial de las aplicaciones informáticas de código libre para promover la implantación de encuestas en línea. Ello ha permitido que en 2011 comenzase a administrarse telemáticamente el cuestionario de evaluación de las actividades formativas de carácter presencial que cubren los docentes mediante un enlace a la encuesta disponible en la aplicación de gestión de la formación de la Escuela. Además, en las actividades de e-learning, tanto el alumnado como el personal docente dan respuesta al cuestionario de satisfacción desde la propia aula virtual en la que se realiza el curso, mientras que el personal dinamizador de las aulas virtuales de la EGAP también cumplimenta una encuesta en línea para realizar el seguimiento del cumplimiento de los compromisos de la Carta de servicios para teleformación.

\section{- UNE-EN ISO 9001:2008}

Un proceso se define como la secuencia de actividades que van añadiendo valor mientras se produce un determinado producto o servicio (modelo EFQM), y la gestión basada en procesos parte de la idea de que se consigue una mayor eficiencia cuando las actuaciones y los recursos se gestionan como un conjunto de actividades mutuamente relacionadas o que interactúan, las cuales transforman elementos de entrada en resultados (ISO 9000, 2005). Este tipo de gestión permite a las organizaciones definir de manera sistemática las actividades que han de realizar, delimitar las responsabilidades, identificar la interrelación entre los procesos, analizar y medir los resultados, centrando su atención sobre éstos, y orientarse, en definitiva, hacia la mejora continua.

17 Informe de avaliación da satisfacción coa formación impartida pola EGAP no ano 2010: Este informe incluye información sobre 393 actividades evaluadas por los alumnos/as, con un total de 9.474 encuestas recabadas, incluyéndose tanto actividades presenciales como de teleformación y está disponible en: <http://egap.xunta.es/fich/nova1539.pdf> (consulta: 30 agosto 2011). 
Partiendo de estos supuestos, a finales de 2010, dio comienzo el proyecto Implantación de un Sistema de gestión de calidad según la norma UNE-EN ISO 9001:2008 en la EGAP, con el doble objetivo de avanzar en el cumplimiento de las expectativas del usuario/a y en la mejora continua de los procesos. En primer lugar, se pusieron en marcha las acciones necesarias para elaborar el informe de diagnóstico de la organización ${ }^{18}$, basado en la revisión documental y la realización de entrevistas en profundidad con los responsables de los distintos servicios de la Escuela, cuyo objetivo fue conocer el actual funcionamiento de la EGAP respecto a los requisitos de la norma UNE-EN ISO 9001:2008. El estudio evidenció la necesidad de documentar las sistemáticas de trabajo de la Escuela, analizar la información existente, establecer procedimientos generales de calidad que den cumplimiento a los aspectos normativos y definir parámetros que permitan medir los niveles de calidad de los diferentes procesos, especialmente en aquellos de carácter crítico, que puedan tener incidencia directa en el nivel de calidad percibido por los grupos de interés de la Escuela.

Paralelamente, se elaboró el Mapa de procesos de la EGAP, en el que se recogen los considerados "estratégicos", "operativos" y "de apoyo"19 para la organización y a partir del cual se está trabajando en la gestión y mejora de los mismos. La selección de los procesos requirió la realización de un análisis destinado a delimitar la estructura funcional de la organización, para lo que fueron tomados en consideración aspectos como su influencia en la misión y estrategia, la incidencia en factores clave, los efectos en la calidad de los servicios o la repercusión en la satisfacción del usuario/a.

En el mapa definido subyacen los principios de la gestión de calidad, como el enfoque al cliente, presente en los procesos relativos a la evaluación de la satisfacción de los usuarios/as, y el liderazgo o el enfoque basado en hechos para la toma de decisiones, en los que se enmarcan los procesos referentes a la planificación y definición de alianzas estratégicas. Los referidos propiamente a la gestión del sistema y la mejora dan respuesta al principio de mejora continua y al de enfoque de sistema para la gestión que se basa en que la comprensión de los procesos interrelacionados como un sistema contribuye a la eficacia y eficiencia de una organización en el logro de sus objetivos. También se desprende del mapa elaborado el principio de participación del personal y el de relaciones mutuamente beneficiosas con el proveedor.

La sesión de presentación del diagnóstico inicial y el mapa de procesos de la organización tuvo lugar a finales del mes de enero de 2011. A lo largo del primer semestre se avanzó también en la documentación de los procedimientos por medio de los cuales se establece la forma de realizar las actividades relacionadas con los procesos

18 Algunas actuaciones son comunes a las desarrolladas en el proyecto de autoevaluación según el Modelo EFQM o la Carta de Servicios de la EGAP: recopilación de documentación, presentación del proyecto, formación del personal, elaboración de un plan de comunicación, etc.

19 Los procesos estratégicos están vinculados a la actividad estratégica de la organización y orientan a esta hacia el cumplimiento de los procesos clave, los procesos operativos se encuentran directamen-

112 la vinculados con la prestación del servicio y en relación directa con el us 
generales de gestión de la organización, siendo identificada una treintena de procedimientos que despliega la EGAP para prestar sus servicios que es preciso documentar para poner en marcha el sistema de calidad, incluyéndose tanto los específicos del área de calidad como aquellos estrechamente relacionados con la prestación del servicio y la cadena de valor de la organización.

La labor de documentación de los procedimientos requiere la participación de los responsables de cada uno de ellos con el objetivo de describir las tareas que incluye y las responsabilidades derivadas de este. Cada procedimiento incorpora la siguiente información: objeto, alcance, definición de los conceptos asociados, responsabilidades concretas de cada uno de los agentes que intervienen, desarrollo detallado y secuencial de las tareas, registros derivados y responsables del archivo, referencias tomadas en consideración para su definición -normativa u otros documentos- y finalmente anexos y formatos ${ }^{20}$ asociados.

Una vez concluida la fase de elaboración de la estructura documental del Sistema de Gestión de la Escuela, dará comienzo la fase de implantación, previa definición de un programa general para su puesta en marcha en el que se establezca el orden en el que es preciso implantar los requisitos del sistema y las actividades a realizar, sus responsables y plazos de ejecución.

\section{El uso intensivo de las TIC: acreditación de competencias digitales, apuesta por la teleformación y servicios digitales en la EGAP}

La segunda línea de transformación que refleja el enfoque integrado de la estrategia de modernización de la EGAP es la introducción de las TIC en la gestión y prestación de sus servicios. En este sentido, la Axenda Dixital de Galicia 2014.gal constituye el marco para la [... ] creación de una cultura en línea con los conceptos de apertura e interoperabilidad como soportes del desarrollo tecnológico, el diseño de sistemas que garanticen el correcto gobierno de las tecnologías de la información y de la comunicación, así como la implantación de sistemas de gestión de la calidad y la seguridad en el ámbito de las tecnologías de la información. Para ello, la Axenda fija una serie de objetivos desarrollados a través de siete líneas estratégicas, que persiguen:

a) Incluir las TIC en todos los ámbitos de los servicios públicos, avanzando desde el concepto de Administración electrónica al de e-Gobierno, y modernizar en profundidad la organización y gestión pública, no sólo lograr su informatización.

b) Adaptar el modelo de Administración a las nuevas posibilidades de gestión, puesto que la incorporación de las TIC contribuye a mejorar la calidad de los

20 Documentos estandarizados para el registro de la información que están asociados a un procedimiento o a una instrucción técnica. 
servicios públicos, a profundizar en la transparencia de su actuación, a promover la participación ciudadana y a garantizar los principios de eficiencia, eficacia, calidad y sostenibilidad.

c) Prestar los servicios públicos transmitiendo seguridad y confianza, asumiendo el reto de la interoperabilidad. El desarrollo de los sistemas de información debe contribuir al intercambio de datos entre las Administraciones y a la reducción de cargas administrativas.

d) Facilitar la vida a la ciudadanía mediante la mejora de los servicios prestados. El avance en el uso de las TIC debe suponer un incremento en el volumen de personas que acceden a ellos, por tanto, un mayor alcance de los mismos, así como la introducción de nuevas funcionalidades en su prestación y, por último, un incremento de su accesibilidad y uso, -por ejemplo, servicios 24x7-.

e) Dinamizar la Sociedad de la Información a través de la formulación y ejecución de políticas que promuevan la inmersión de las TIC en la sociedad y el acceso a la tecnología por parte de todos los agentes sociales, para lo que resulta imprescindible que la ciudadanía tenga las competencias digitales y la motivación necesarias para participar activamente en su desarrollo.

\section{- Acreditación de competencias digitales}

En este escenario se hace necesario que los empleados públicos adquieran también competencias digitales que contribuyan a desarrollar estas líneas estratégicas. En concreto, la línea 3 de la Axenda dixital, Ciudadanía digital, incorpora el proyecto de Competencias Dixitais en Ofimática de Galicia que contempla tanto un itinerario formativo para los empleados públicos -Plan Ofimático de Galicia- como la realización de pruebas específicas de libre acceso.

El objetivo que se recoge en el PEG es que el 100\% del funcionariado tenga competencias digitales en 2014, y para ello se ha publicado recientemente el Decreto 218/2011, de 17 de noviembre, por el que se regula la certificación gallega de competencias digitales en ofimática, que establece que las tecnologías de la información y las comunicaciones (TIC) [...] deben servir como catalizadoras de los ejes marcados en el Plan Estratégico, además de como mecanismos facilitadores de la cohesión social, de la calidad de vida de la ciudadanía gallega y de la generación de empleo de calidad, impulsando, asimismo, una administración austera, eficiente y próxima a la ciudadanía. [... ] Por medio del presente decreto, y en desarrollo de la línea estratégica L3: Ciudadanía digital, en su punto L3-P3: Programa de acreditación de competencias digitales, de la Agenda Digital 2014.gal, se crea la certificación gallega de competencias digitales en ofimática. Esta certificación tiene como objetivo fundamental el fomento

114 del uso de las tecnologías de la información y las comunicaciones mediante el apoyo y 
reconocimiento de los esfuerzos realizados para la adquisición de capacidades y aptitudes en el ámbito de las tecnologías de la información y las comunicaciones.

\section{GRÁFICO 5}

Enfoque Lifelong Learning: Los ciudadanos gallegos como nativos digitales del futuro

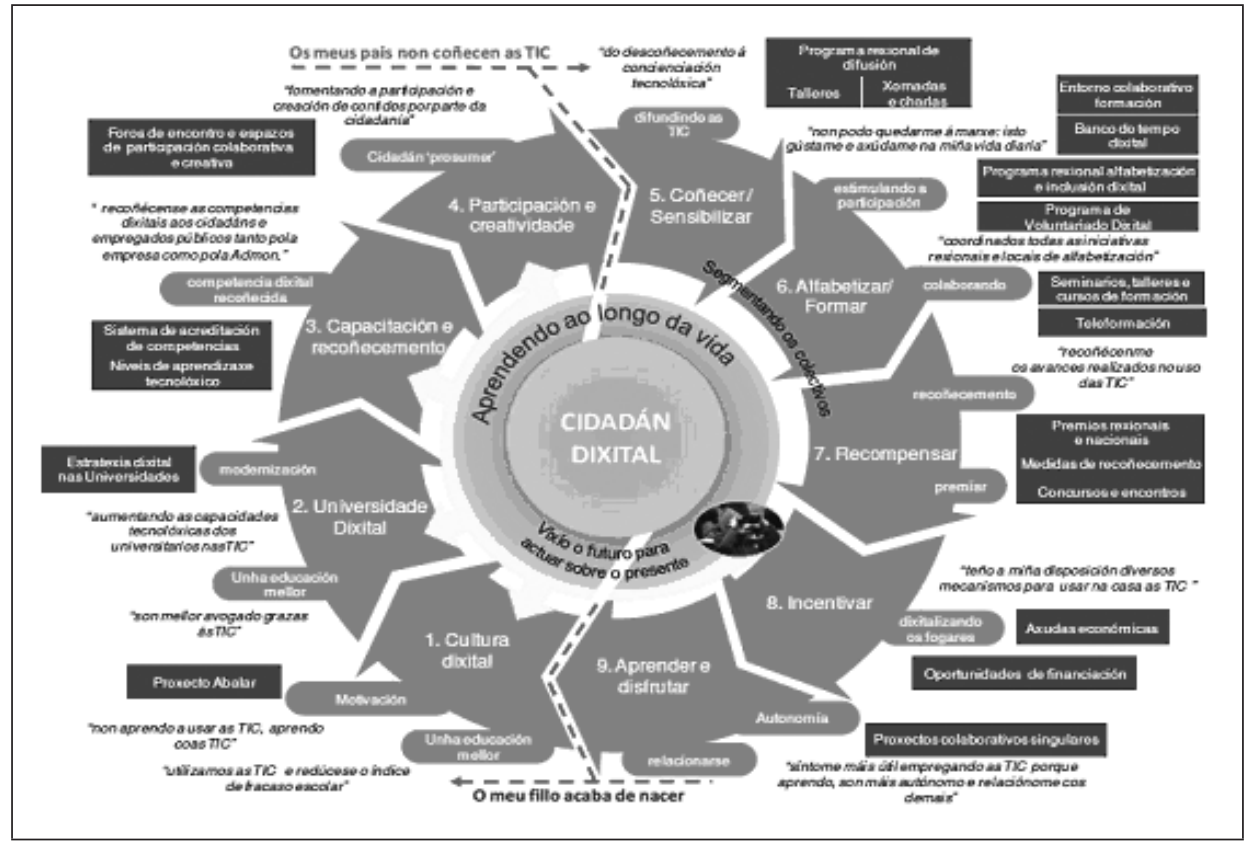

Fuente: Resumen Ejecutivo de la Axenda Dixital Galicia 2014.org.

La EGAP desempeña un papel fundamental en este contexto al ser la responsable, en estrecha colaboración con la SXMIT, tanto de los cursos de formación que son parte del Plan Ofimático de Galicia, dirigidos a los empleados públicos de la Administración de la Comunidad Autónoma de Galicia, como de las pruebas de libre acceso, un aspecto, este último, que le permite liberar plazas en favor de aquellas personas que realmente necesitan formación y no sólo su reconocimiento formal.

De este modo, los certificados que acreditan tener superadas actividades formativas integradas en el Plan Ofimático, expedidos por la EGAP, constituyen una de las vías oficiales establecidas en el decreto (art. 6.2.c) para obtener la certificación gallega de competencias digitales en ofimática, como mecanismo normalizado de certificación por parte de la administración pública que garantiza la calidad de los conocimientos, destrezas y capacidades que las personas precisan en el manejo de aplicaciones informáticas en la gestión de la información y la documentación.

Para obtener la certificación de competencias TIC, cada persona tendrá que realizar un itinerario formativo compuesto por cinco cursos (190 horas) cuya superación 
permite acreditar las competencias digitales en ofimática del personal al servicio de la administración pública de la Comunidad Autónoma de Galicia.

\section{CUADRO 3}

Convocatoria 2011 para la certificación de competencias TIC ${ }^{1}$

\begin{tabular}{|c|c|c|c|c|}
\hline \multicolumn{2}{|c|}{ CONVOCATORIA 2011} & \multirow{2}{*}{$\frac{\text { Ediciones }}{7}$} & \multirow{2}{*}{$\begin{array}{c}\text { Plazas } \\
350\end{array}$} & \multirow{2}{*}{$\begin{array}{c}\text { Horas } \\
210\end{array}$} \\
\hline Sistema operativo, búsqueda de & Linux & & & \\
\hline correo electrónico & Windows 7 & 7 & 350 & 210 \\
\hline \multirow{2}{*}{$\begin{array}{l}\text { Aplicaciones informáticas de } \\
\text { tratamiento de textos }\end{array}$} & OpenOffice & 7 & 350 & 210 \\
\hline & Microsoft Office & 7 & 350 & 210 \\
\hline \multirow{2}{*}{$\begin{array}{l}\text { Aplicaciones informáticas de hojas } \\
\text { de cálculo }\end{array}$} & OpenOffice & 7 & 350 & 350 \\
\hline & Microsoft Office & 7 & 350 & 350 \\
\hline \multirow{2}{*}{$\begin{array}{l}\text { Aplicaciones informáticas de bases } \\
\text { de datos relacionales }\end{array}$} & OpenOffice & 7 & 350 & 350 \\
\hline & Microsoft Office & 7 & 350 & 350 \\
\hline \multirow{2}{*}{$\begin{array}{l}\text { Aplicaciones informáticas de } \\
\text { presentaciones gráficas }\end{array}$} & OpenOffice & 7 & 350 & 210 \\
\hline & Microsoft Office & 7 & 350 & 210 \\
\hline \multicolumn{2}{|l|}{ TOTAL } & 70 & 3.500 & 2.660 \\
\hline
\end{tabular}

Fuente: Unidad de Investigación, Evaluación y Calidad de la EGAP.

La EGAP, para poder realizar esta convocatoria en 2011, desarrolló los contenidos multimedia de estas actividades garantizando su adaptación al Catálogo Nacional de Cualificaciones Profesionales. Este proyecto, desarrollado en colaboración con la SXMIT, contribuirá a la modernización de la administración y aportará un valor añadido a los servicios que se presta a la ciudadanía.

\section{- Apuesta por la teleformación}

La EGAP quiere dar un paso más allá en el impulso a la teleformación mediante la mejora de todos los aspectos relacionados con ésta: contenidos, dinamización de los entornos de aprendizaje, capacitación de los profesionales, mejora continua de los procesos de gestión de las actividades, etc. De este modo, la Escuela se alinea nuevamente con los objetivos de la Axenda Dixital de Galicia 2014.gal que persigue impulsar la inclusión de Galicia en el nuevo contexto digital europeo y la convergencia con el escenario normativo definido por el Decreto 198/2010, de 2 de diciembre, por el que se regula el desarrollo de la Administración electrónica en la Xunta de Galicia y en las entidades de ella dependientes, dirigido a fomentar un uso intensivo de los medios electrónicos por parte de la Xunta.

21 Resolución de 10 de mayo de 2011 por la que se convocan varios cursos de formación continua para el personal al servicio de la Administración pública de la Comunidad Autónoma de Galicia (Diario Ofi-

116 cial de Galicia de 13 de mayo de 2011). 
En los últimos años, la EGAP ha realizado una decidida apuesta por la incorporación de las TIC al proceso formativo con el objetivo de aprovechar al máximo las ventajas de la sociedad de la información y garantizar la prestación de un servicio acorde con las necesidades y expectativas de sus usuarios/as. En este sentido, la teleformación permite acercar la formación a un mayor número de personas, evita los desplazamientos y constituye un instrumento de primer orden para la conciliación de la vida personal y profesional, al permitir el acceso a los entornos y recursos formativos las 24 horas, los 365 días del año, sin límites temporales ni territoriales. Por eso, desde la Escuela, mediante la apuesta por la teleformación, se pretende impulsar una administración más abierta, flexible, próxima y comprometida con la ciudadanía.

Los cursos de teleformación que ofrece la Escuela se desarrollan a través de un Entorno Virtual de Aprendizaje (EVA) al que se accede a través de la página web de la $\mathrm{EGAP}^{22}$. Cada aula virtual está diseñada para que el alumnado pueda llevar a cabo un proceso de autoformación y autoaprendizaje en función de su disponibilidad de tiempo y de sus necesidades, con independencia del lugar donde se encuentre. Esta modalidad posibilita que la oferta formativa de la EGAP se distribuya de forma más equilibrada en todo el territorio gallego, caracterizado por la dispersión territorial. Además, en la plataforma de teleformación, los cursos cuentan con herramientas y materiales destinados al adecuado desarrollo del proceso formativo que favorecen la interactividad (tareas, cuestionarios y ejercicios prácticos que permiten la retroalimentación casi en tiempo real) y el aprendizaje colaborativo (foros, chats y mensajería interna).

Finalmente, además de las ventajas en términos de flexibilidad y conciliación, la teleformación contribuye a realizar un uso más eficiente de los recursos por parte de la Escuela, posibilitando ofrecer formación a un mayor número de personas y a un menor coste.

La calidad del proceso formativo en la modalidad de teleformación trata de garantizarse a través de dos mecanismos: el cumplimiento de los compromisos que se recogen en la Carta de servicios de la EGAP y el análisis de la satisfacción con la acción formativa (cuestionarios para la evaluación de acciones formativas de e-learning). En relación a esta cuestión, cabe señalar que, a pesar de la novedad y de las posibles reticencias que la modalidad de teleformación pueda generar a priori entre una parte del alumnado no habituada al empleo de las TIC en la formación, los niveles de satisfacción de los usuarios/as atendiendo a las distintas modalidades formativas -presencial y teleformación- son muy similares (4,3 en ambos casos, en una escala de 1 a 5) ${ }^{23}$.

En relación a esta iniciativa, hay que destacar también que la experiencia y la formación específica del personal de la Escuela para la gestión y dinamización de las

22 Se puede acceder a la plataforma de teleformación de la EGAP en: http://egap.xunta.es/teleformacion.php>.

23 Informe de avaliación da satisfacción coa formación impartida pola EGAP no ano 2010, disponible en: <http://egap.xunta.es/fich/nova1539.pdf> (consulta: 30 agosto 2011). 
acciones de teleformación han contribuido a un incremento de la oferta formativa on line, permitiendo avanzar hacia el objetivo de la EGAP, incluido en el PEG 2010-2014, de ofrecer el $60 \%$ de la formación en esta modalidad.

\section{GRÁFICO 1}

Evolución de la oferta de plazas según modalidad de formación

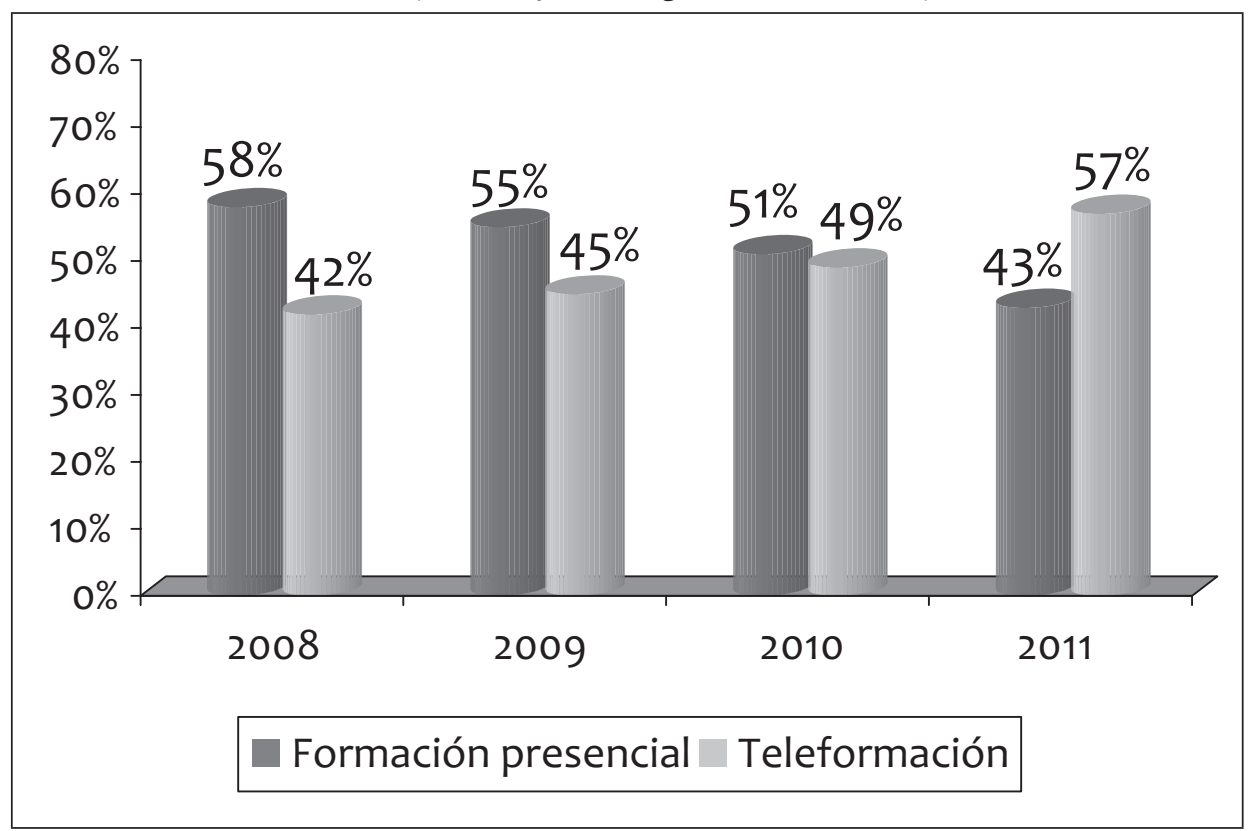

Fuente: Unidad de Investigación, Evaluación y Calidad de la EGAP.

El incremento de la oferta formativa en la modalidad de teleformación ha requerido, por lo tanto, la implicación y formación específica del personal de la EGAP como dinamizadores de teleformación, al objeto de desarrollar la gestión de estos cursos en un entorno virtual. De este modo, los dinamizadores o gestores se encargan de asegurar el acceso al aula por parte de todas las personas matriculadas y sirven de interlocutores entre los tutores de los cursos, el alumnado y el personal de administración de la EGAP, velando, en todo momento, por la calidad y la satisfacción con el desarrollo del curso en el entorno virtual.

\section{- Los servicios digitales de la EGAP}

Desde hace varios años, la EGAP ha ido conformando toda una infraestructura informática para la gestión integral de su actividad. De esta manera nace Egapolis, una base de datos con información relativa a la actividad de la Escuela desde 1991, consistente en una aplicación web que se ha ido configurando paulatinamente, a tra-

118 vés del desarrollo de diversos módulos, en función de las necesidades de la organiza- 
ción ${ }^{24}$. Esta aplicación articula tanto la información y comunicación interna, entre el personal de la Escuela, como externa, con los usuarios/as de los servicios de la EGAP.

Destacan los módulos de solicitud de cursos "Zona de matrícula" (aspecto que recibe unas extraordinarias valoraciones por parte del alumnado), "reserva de aulas" o "Actividades hoxe" que da a conocer la actividad diaria de la EGAP. Para la comunicación interna entre el personal de la Escuela se ha desarrollado un módulo de incidencias y mensajería. Por otra parte, la comunicación de los usuarios/as con la EGAP se articula mediante la habilitación de formularios web, a través de los que puede plantearse una duda, comentario o queja a los distintos servicios o unidades de la Escuela, así como renunciar a una actividad formativa o realizar la solicitud de reserva de un aula.

A través de la página web de la EGAP, y de forma coordinada con Egapolis, los distintos usuarios/as -internos y externos- pueden acceder a los servicios anteriormente descritos así como a otras bases de datos -como el "Rexistro de formadores"- o a la propia "Plataforma de teleformación" de la EGAP.

En este contexto, un aspecto fundamental para la Escuela es la interoperabilidad, referida en este caso al traspaso de los datos relativos a la formación a la Dirección Xeral da Función Pública para que proceda a la actualización de los expedientes de los empleados públicos. A este respecto, la EGAP asume en su carta de servicios el compromiso concreto de Impulsar la interoperabilidad entre los distintos Servicios, a través del intercambio de datos e información con Función Pública, remitiéndoles diariamente la información relativa a las actividades realizadas por el personal al servicio de la Administración pública de la Comunidad Autónoma, para que sea incorporada a su expediente formativo y esté permanentemente actualizado y accesible a las personas interesadas a través del Portal do Empregado Público (Portax) (Compromiso 4.6 de la Carta de servicios de la EGAP).

Otros servicios digitales que ofrece la EGAP a través de su página web son la consulta y descarga gratuita de sus publicaciones: temarios, revistas e informes. Además, la biblioteca de la Escuela está inserta en la red de bibliotecas de Galicia, siendo posible acceder electrónicamente a su catálogo en: <http://egap.rbgalicia.org/>.

Por otra parte, con la aprobación del Decreto 198/2010, de 2 de diciembre, por el que se regula el desarrollo de la Administración electrónica en la Xunta de Galicia y en las entidades de ella dependientes, se da un nuevo impulso a la digitalización y homogeneización de los servicios electrónicos que prestan las entidades de la Xunta, cuya implementación se canaliza a través del Plan de Modernización de la Administración pública ${ }^{25}$.

24 Para la mejora de las herramientas de gestión de la formación, se constituyó un grupo de trabajo específico en el seno de la organización integrado por personal de las distintas áreas departamentales.

25 Disponible en: <http://imit.xunta.es/portal/desenvolvementoegoberno/administracionelectronica/ plan_de_modernizacion/ambitoactuacion.html> (consulta: 9 agosto 2011). 
En este contexto, además del empleo de las TIC en la gestión de la actividad inherente a la Escuela, la EGAP se ha sumado al proyecto de desarrollo de la Administración electrónica de la Xunta, integrando en la organización los distintos sistemas y servicios digitales de carácter horizontal de la Xunta de Galicia: el correo electrónico corporativo, la aplicación de envío de normas para su publicación en el Diario Oficial de Galicia, el sistema de información de gestión contable (Xumco), el sistema electrónico de facturación, el sistema de información de nóminas o el acceso a la plataforma de contratación.

Por último, la prestación de estos servicios digitales está acompañada de dos elementos de gran relevancia como son la protección de datos y la accesibilidad. En este sentido, la EGAP, en cumplimiento de la Ley orgánica 15/1999, de protección de datos de carácter personal, cuenta con una política y protocolos específicos de protección de datos, al tiempo que ha considerado los aspectos formales de accesibilidad de su página web ${ }^{26}$.

\section{CONCLUSIONES}

Las etapas del proyecto modernizador de la EGAP se ajustan a las fases del proceso de cambio organizativo (Ramió, 1996: 219) de percepción de la necesidad de cambio, planificación o diseño del cambio, ejecución, control del proceso y evaluación. La autoevaluación de la EGAP según el Modelo EFQM actuó como catalizador del proceso de reforma al poner de manifiesto las principales acciones que debería acometer la Escuela y maximizar al mismo tiempo las dinámicas de interacción entre los agentes participantes, contribuyendo a simplificar la implementación de los cambios y a minimizar las eventuales disfunciones en el proceso de implantación.

Además del conjunto de acciones de mejora desarrolladas, la ejecución jurídica o normativa del cambio se plasmó en el Decreto 175/2010, de 14 de octubre, por el que se establece la estructura orgánica de la EGAP. El control del proceso de transformación se lleva a cabo a través de sistemas de indicadores desarrollados en el marco de las distintas acciones de mejora, en tanto que, en último término, está previsto cerrar el ciclo mediante la evaluación del cambio, que tendrá lugar, una vez desplegados los distintos proyectos de mejora, con la segunda autoevaluación de la EGAP según el Modelo EFQM de excelencia.

El análisis de las distintas actuaciones que componen el proyecto modernizador de la EGAP permite esbozar posibles hipótesis de estudio para próximos análisis sobre el impacto de la implementación conjunta de las líneas de transformación calidad y TIC en el desarrollo de proyectos de cambio y modernización administrativa. La experiencia de la EGAP pone de manifiesto, en consonancia con el marco normativo de modernización de la administración, cómo el desarrollo paralelo de acciones en materia de calidad y nuevas tecnologías alberga un enorme potencial para gestionar los

26 Véase: <https://egap.xunta.es/accesibilidad.php> (consulta: 9 agosto 2011). 
servicios de manera eficiente y dar una respuesta satisfactoria a los usuarios/as, debido a las posibilidades que ofrece para la innovación en la gestión.

El avance en el uso de las nuevas tecnologías ha de producirse, en cualquier caso, en el marco de un sistema o conjunto de transformaciones organizativas que interaccionan y se refuerzan mutuamente en el seno de un proyecto integral de modernización, que incluye modificaciones en las estructuras organizativas y en los modelos de gestión. Así, frente a eventuales acciones concretas y aisladas con escasa incidencia en la mejora global de la organización, la innovación en la gestión que aporta la combinación de calidad y nuevas tecnologías constituye un motor para el cambio organizativo a distintos niveles -esto es, en el plano de los resultados, de los procesos y de la propia configuración de la organización-.

El desarrollo simultáneo de acciones en materia de calidad y TIC, además de configurarse como una vía para la innovación en la gestión, ha ayudado en el caso de la EGAP a potenciar la orientación al usuario/a y el desarrollo de nuevos cauces de interacción entre la administración y los destinatarios de sus servicios. A este respecto, la implementación de la propia estrategia modernizadora y la participación e implicación del personal de la Escuela han contribuido a generar una cultura organizativa caracterizada por la orientación al usuario/a, cuyas demandas se han visto modificadas sustancialmente en los últimos años a la luz de los cambios sociales y del desarrollo de la sociedad del conocimiento.

Por tanto, la calidad y las TIC no constituyen un fin en sí mismas, sino un medio que posibilita poner en marcha un nuevo modelo de gestión y una relación distinta entre la administración y los destinatarios de sus servicios. Esta estrategia bifronte se desprende de la propia visión de la Escuela, que en el marco de este proceso se ha visto modificada para reforzar su papel como organización del conocimiento, al hacer referencia a que la EGAP incorpora las nuevas tecnologías en la gestión del conocimiento y presta sus servicios con la máxima calidad.

En definitiva, la EGAP ha puesto en marcha una actuación integral de modernización administrativa, en la que las distintas líneas de mejora emprendidas, basadas en la calidad y en el uso intensivo de las nuevas tecnologías, se retroalimentan y refuerzan mutuamente, en línea con el marco normativo vigente. De este modo, la Escuela muestra cómo la combinación de estas dos dimensiones está presente en cada uno de los proyectos a través de los que despliega su estrategia, contribuyendo no sólo a la satisfacción de los usuarios/as y a la provisión de servicios de máxima calidad, sino también a un proceso más amplio de transformación hacia una administración más transparente, más participativa, más eficiente y más responsable.

\section{REFERENCIAS BIBLIOGRÁFICAS Y DOCUMENTALES}

Ballart, X. y Ramió, C. (2000), Ciencia de la Administración. Valencia: Tirant lo Blanch. Brugué, Q. y Subirats, J. (1996), Lecturas de gestión pública. Madrid: MAP. 
Cerrillo i Martínez, A. (2005), La gobernanza hoy: 10 textos de referencia. Madrid: INAP. Cerrillo i Martínez, A. (2010), “La administración electrónica en el derecho autonómico comparado”, Revista Vasca de Administración Pública, 86: 15-60.

Cerrillo i Martínez, A. (2011), “CCómo facilitar el ejercicio de los derechos de los ciudadanos en la Administración Electrónica?", Revista de Internet, Derecho y Política, 12: 31-43. Disponible en (Consulta: 6 diciembre 2011).

Consellería de Facenda (2010), Plan Estratéxico de Galicia 2010 - 2014. Santiago de Compostela: Xunta de Galicia. En línea: < http://www.conselleriadefacenda.es/ plan-estratexico/pdfs/plan_estratexico_version_web.pdf> (Consulta: 9 agosto 2011).

Consellería de Presidencia, Administracións Públicas e Xustiza (2008), Guía descriptiva de elaboración de Cartas de Servicios en la Xunta de Galicia. Santiago de Compostela: Xunta de Galicia. En línea: <http://cpapx.xunta.es/c/document_library/ get_file?file_path=/portal-cpapx/DXAvalidacionReforma/FerramentasCartasServizos/CAST_cartas_de_servicio_vinculo.pdf> (consulta: 9 agosto 2011).

Criado, J.L. (2007), "A europeización dos sistemas administrativos e as políticas públicas. Unha aproximación aos mecanismos institucionais na e-administración”, Administración \& Cidadanía, 3(2): 95-113.

Escola Galega de Administración Pública (2010). Informe da avaliación da satisfacción coa formación impartida pola EGAP no ano 2010. Santiago de Compostela: EGAP. En línea: <http://egap.xunta.es/fich/nova1539.pdf> (Consulta: 30 agosto 2011).

European Foundation for Quality Management (2010), EFQM Excellence Model 2010. Belgium: EFQM. En línea: <http://www.efqm.org> (consulta: 17 diciembre 2011).

Ferlie, E, Lynn, L.E. y Pollitt, C. (2005), The Oxford handbook of public management. Oxford: Oxford University Press.

Friedman, J. (2001), Planificación en el ámbito público. Madrid: INAP.

Grau, M. y Mateos, A. (2002), Análisis de políticas públicas en España: enfoques y casos. Valencia: Tirant lo Blanch.

Grote, J. R. y Gbikpi, B. (2002), Participatory Governance. Political and Societal Implications. Opladen: Leske \& Budrich.

Grupo Observatorio de la Administración Electrónica de las Comunidades Autónomas (2010), Informe CAE 2009. La Administración Electrónica en las Comunidades Autónomas. Madrid: Centro de Publicaciones del Ministerio de Política Territorial y Administración Pública. En línea: <http://administracionelectronica.gob.es/recursos/pae-000005852.pdf> (consulta: 6 diciembre 2011).

Kooiman, J. (2005), “Gobernar en gobernanza”, en A. Cerrillo i Martínez, La gobernanza hoy: 10 textos de referencia. Madrid: INAP.

Lindblom, C.E. (1991), El proceso de elaboración de políticas públicas. Madrid: MAP.

López Casanovas, G. (2003), “Los nuevos instrumentos de la gestión pública”. Colección Estudios Económicos "La Caixa”, 31. En línea: <http://www.pdf.lacaixa.comunicacions.com/ee/esp/ee31_esp.pdf> (Consulta: 28 agosto 2011).

Maderuelo Fernández, J.A. (2002), "Gestión de la calidad total. El modelo EFQM de 122 excelencia”, MEDIFAM 2002; 12(10): 631-640. 
Mayntz, R. (2000), "Nuevos desafíos de la teoría de Governance”, Instituciones y Desarrollo: desarrollo institucional para una gobernabilidad democrática, 7: 35-52

Membrado Martínez, J. (1999), La gestión empresarial a través del modelo europeo de excelencia de la E.F.Q.M. Madrid: Díaz de Santos.

Ministerio de Política Territorial y Administración Pública (2010), Informe CAE 2009, la administración electrónica en las Comunidades Autónomas. Madrid: Portal de administración electrónica. En línea: <http://administracionelectronica.gob.es/> (consulta: 7 diciembre 2011).

Pérez Sánchez, M. (2005), Análisis de políticas públicas. Granada: Universidad de Granada.

Peters, B. G. (2006), “Democracia e administración pública: a conexión emerxente”, Administración \& Cidadanía, 1: 29-46.

Pollitt, C. y Bouckaert, G. (2004), Public Management Reform. A comparative analysis. New York: Oxford University Press.

Possenti, V. (1985), "La sociedad abierta en el pensamiento del siglo XX (Bergson, Popper, Maritain)”, Diálogo filosófico, 3: 283-290.

Prats i Catalá, J. (2005), De la burocracia al management, del management a la gobernanza, Madrid: INAP.

Ramió, C. (1996), Teoría e práctica do cambio organizativo na Administración Pública. Santiago de Compostela: EGAP

Ramió, C. (1999), Teoría de la Organización y Administración Pública. Madrid: Tecnos.

Sabatier, P. A. (1999), Theories of the policy process. Boulder: Westview Press.

Simon, H.A. (1997), Administrative behaviour: a study of decision-making processes in administrative organizations. Nueva York: The Free Press.

Subirats, J. (1994), Análisis de políticas públicas y eficacia de la Administración. Madrid: Ministerio de Administraciones Públicas.

Voces, C. Rodríguez, A. y Caínzos, M. (2009), Estudo da satisfacción dos cidadáns galegos cos servizos públicos 2007. Santiago de Compostela: EGAP.

Xunta de Galicia (2010), Axenda Dixital de Galicia 2014.gal. Santiago de Compostela: Iniciativas de Modernización e Innovación Tecnolóxica. En línea: <http://imit.xunta.es/portal/documentos/docexecutivoaxendadixital.html?__locale=es> (Consulta: 9 agosto 2011).

Xunta de Galicia (2010). Plan de modernización de la Administración Pública en Galicia. Santiago de Compostela: Iniciativas de Modernización e Innovación Tecnolóxica. En línea: <http://imit.xunta.es/portal/desenvolvementoegoberno/administracionelectronica/plan_de_modernizacion/ambitoactuacion.html> (Consulta: 9 agosto 2011$)$. 